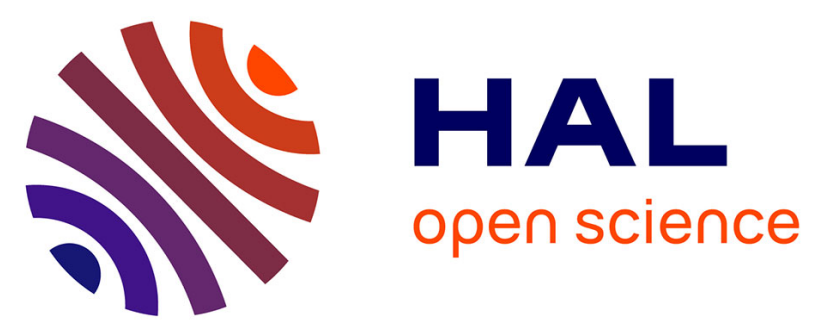

\title{
Learning How to Generate Kinesthetic Motor Imagery Using a BCI-based Learning Environment: a Comparative Study Based on Guided or Trial-and-Error Approaches
}

Sébastien Rimbert, Laurent Bougrain, Stéphanie Fleck

\section{To cite this version:}

Sébastien Rimbert, Laurent Bougrain, Stéphanie Fleck. Learning How to Generate Kinesthetic Motor Imagery Using a BCI-based Learning Environment: a Comparative Study Based on Guided or Trialand-Error Approaches. SMC 2020 - International IEEE Conference on Systems, Man and Cybernetics, Oct 2020, Toronto, Canada. hal-02920306

\author{
HAL Id: hal-02920306 \\ https://hal.inria.fr/hal-02920306
}

Submitted on 24 Aug 2020

HAL is a multi-disciplinary open access archive for the deposit and dissemination of scientific research documents, whether they are published or not. The documents may come from teaching and research institutions in France or abroad, or from public or private research centers.
L'archive ouverte pluridisciplinaire HAL, est destinée au dépôt et à la diffusion de documents scientifiques de niveau recherche, publiés ou non, émanant des établissements d'enseignement et de recherche français ou étrangers, des laboratoires publics ou privés. 


\section{Learning How to Generate Kinesthetic Motor Imagery Using a BCI-based Learning Environment: a Comparative Study Based on Guided or Trial-and-Error Approaches}

\author{
$1^{\text {st }}$ Sébastien Rimbert \\ Neurorhythms team \\ Université de Lorraine, CNRS, Inria \\ LORIA, F-54000 Nancy, France \\ sebastien.rimbert@inria.fr
}

\author{
$2^{\text {nd }}$ Laurent Bougrain \\ Neurorhythms team \\ Université de Lorraine, CNRS, Inria \\ LORIA, F-54000 Nancy, France \\ laurent.bougrain@loria.fr
}

\author{
$3^{\text {rd }}$ Stéphanie Fleck \\ PErSEUs \\ Université de Lorraine \\ F-57073 Metz, France \\ stephanie.fleck@univ-lorraine.fr
}

\begin{abstract}
Kinesthetic Motor Imagery (KMI) is a mental task which, if performed properly, can be very relevant in sports training or rehabilitation with a Brain-Computer Interface (BCI). Unfortunately, this mental task is generally complex to perform and can lead to a high degree of variability in its execution, reducing its potential benefits. The reason why the task of KMI is so difficult to perform is because there is no standardized way of instructing the subject in this mental task. This study presents an innovative BCI called Grasp-IT thought to support the learning of the KMI task, and the evaluation of two different learning methods: (i) a first one guided by an experimenter and based on the notion of progressiveness and (ii) a second one where the learners are alone and practice by trial and error. Our findings based on EEG analyses and subjective questionnaires validate the design of the Grasp-IT BCI and opens up perspectives on KMI learning modalities.
\end{abstract}

Index Terms-Kinesthetic Motor Imagery; Brain-Computer Interface; Grasp-IT; Stroke Rehabilitation; BCI learning environment; Human-Computer Interaction

\section{INTRODUCTION}

Currently, synaptic plasticity resulting from the practice of kinesthetic motor imagery (KMI) opens up promising fields, particularly to optimize athletes' training or motor recovery after stroke [1]. KMI is a mental process which can be described as the ability to imagine performing a movement without executing it, specifically by reactivating the haptic sensations (i.e., tactile, proprioceptive, and kinesthetic) felt during a real movement [2]. Because a KMI corresponds to the recall of a motor act, this mental task activates the primary motor cortex and the additional motor areas in the same way as a real movement would do [3].

Performing a KMI generates changes in the cerebral activity that can be recorded by the electroencephalographic (EEG) technique. In particular, the power of the sensorimotor rhythms (mu/alpha and beta) is modulated when executing a KMI. Before and during a KMI, a gradual decrease in power in the $\mathrm{mu} / \mathrm{alpha}(7-13 \mathrm{~Hz})$ and beta $(15-30 \mathrm{~Hz})$ bands is observed [4], while the end of the KMI typically results in an increase in power in the beta band [5] and occasionally also in the mu band [6]. Power modulations in the sensorimotor rhythms can thus be detected and used as a modality of human-computer interaction, then called brain-computer interface (BCI). BCIs have many applications, but the use of KMI-based BCIs for post-stroke rehabilitation has grown considerably since several years [7].

Unfortunately, several pieces of evidence show that the KMI task is generally complex to perform [8]. This complexity can lead to highly variable KMI-based BCI results [9], degrading the quality of the interaction, and consequently limit the potential benefits sought (e.g., motor rehabilitation). However, the inner nature of this mental task is, in part, responsible for the difficulties experienced by the users. Indeed, people are faced with two important difficulties. Firstly, the KMI remains intangible (i.e., not graspable intellectually and physically) which limits the understanding of how to properly perform a KMI. In fact, in BCI protocols, the subject rarely (or in an unstandardized manner) receives the practical way in which she/he has to perform the task. Secondly, the KMI does not generate the proprioceptive feedback usually perceived during a real movement. These two reasons could explain why some people have difficulties in perceiving their level of performance, and therefore in making progress.

Considering the hopes and challenges associated with BCI technology and motor rehabilitation [7], developing approaches that improve the implementation of the KMI task by maintaining a quality user experience is essential [10], [11]. We therefore consider that the first challenge is to better instruct the KMI task and support its implementation. Echoing [12], we believe that gradual KMI instruction would help inexperienced subjects to learn it properly. Through an interdisciplinary approach, we designed a new KMI-based BCI called Grasp-IT thought to provide a KMI-based interaction which support the KMI 


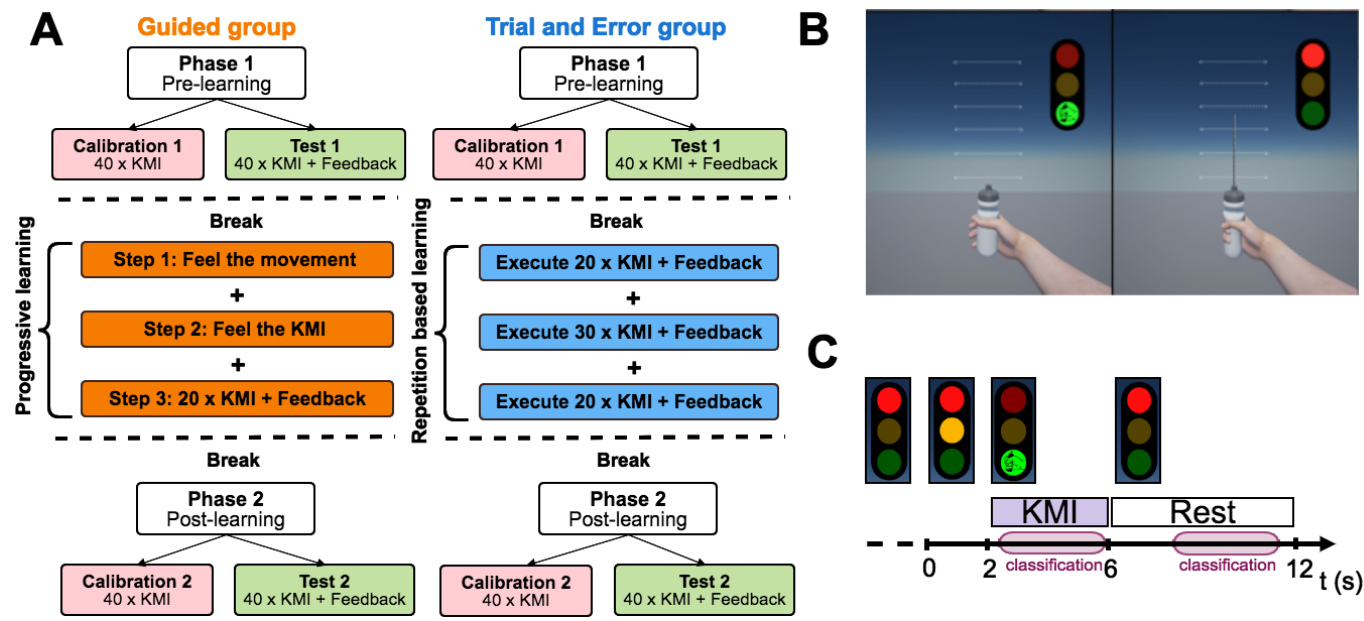

Fig. 1. (A) First phase including both a calibration and a test steps. One group $(\mathrm{n}=17)$ benefited from progressive learning guided by an instructor and with Grasp-IT (called Guided Group). A second group ( $\mathrm{n}=20)$ learned KMI intuitively by repeating the KMI task with Grasp-IT (called Trial-\&-Error Group). All the participants finally completed a second phase, which again consisted of a calibration and a test steps. (B) The non-immersive virtual environment used to deliver cues and feedback. (C) Timing scheme for each trial: the subject was in a resting state when the light was red. The participant performed a right-hand KMI during $4 \mathrm{~s}$ when the light was green. We segmented each trial into a kinesthetic time for classification (KTC) of $3.5 \mathrm{~s}$ and a rest time for classification (RTC) of $3.5 \mathrm{~s}$.

learning and the associated user experience (UX) [13]. Inspired by the fact that motor skills developed during activities, such as sports, music, etc., seem to facilitate the performance and would improve the detection of KMI by a BCI [9], [14], the Grasp-IT BCI is designed to support the learning process through the implementation of a KMI for a day-to-day gesture corresponding to a right-hand grasping movement.

In this study, we assessed the impact of a short-term learning protocol on the learner's effectiveness in KMI implementation immediately afterwards. In one condition the subjects were progressively guided to figure out the KMI allowing to interact with Grasp-IT ( $\mathrm{n}=17$ participants). In the second condition ( $\mathrm{n}=20$ participants), they have to learn by themselves how to produce KMI interacting with Grasp-IT. We also assessed the design choices of the Grasp-IT BCI and the perceived quality of the learning experience. To estimate task mastery, possible performance differences, and potential UX influencing factors, we studied ERD/ERS modulations during the task, online classification accuracy, and UX questionnaire results, each recorded or collected before and after the two learning approaches.

\section{Material And Methods}

This study focuses on two major issues: (i) assess the Grasp-IT design thought to support the KMI learning and (ii) evaluate the relevance of a new progressive and guided KMI learning protocol, compared to a trial-and-error learning conducted alone, on the ability to perform in kinesthetic motor imagery.

\section{A. BCI design}

The Grasp-IT environment (for "Grasp a KMI in an Interactive and Tangible manner") aims to make KMIs more tangible, understandable and easy to implement for first-time use [13]. Grasp-IT is a BCI designed according to three main criteria that we believe conducive to a positive and effective learning experience:

- The affordance effect of a common object (i.e., the implicit knowledge of its use) to improve comprehension of the task and enable spontaneous execution, and then provide a better stimulation of the sensorimotor cortex [15]. Indeed, the Grasp-IT non-immersive virtual environment (ni-VR) is designed to promote a Goal Oriented Imagery task [16]. Hence, Grasp-IT should invite the user to imagine squeezing a bottle she/he really has in her/his hand as if she/he wants to produce a powerful water jet, helped by a similar bottle also visible on the first-person view in the ni-VR environment (Fig. 1B).

- Visual cues designed to help users focus alone on the KMI task and execute it at the right time. Indeed, the ni-VR environment of the system provides visual cues via a traffic light, allowing the subject to easily identify when to perform KMI (green light) and rest (red light) tasks. An amber light also instructs users to prepare themselves before having to perform the KMI, ensuring that the subject could be fully relaxed during the rest task and to avoid motor preparation during the resting state.

- A situation presenting a challenge and feedback to selfevaluate one's success in the BCI task, which is crucial to enable users to measure their performance and seek to progress, and thus maintain motivation [17]. This feedback presents the levels of success achieved, associated with BCI performance through the amplitude of the water jet, which is proportional to the intensity of the cerebral electrical signal generated by the user. This feedback is designed to guide the user's concentration and strategy in order to provide the most 
effective stimulation of the motor cortex.

\section{B. Learning protocol}

To improve the ability to perform the KMI, we designed two different learning strategies: (i) the first one based on progressiveness and guided by an instructor (Guided group) and (ii) the second one based on trial-and-error learning by task repetition (Trial-\&-Error group) (Fig. 1A).

For the guided group, we offer a step-by-step protocol, designed to support learners better understand how to control a KMI-Based BCI, based on three different gradual steps:

1) Feel the movement: according to the literature linked with meditation benefits [18], this first step focuses on increasing the participant's awareness of all the perceptions present during the actual performance of the expected motor task. In this stage, the instructor first described all the sensations that could be felt during the movement (e.g., sensations of pressure and heat, muscle contraction, skin perceptions, activation of the peripheral nerve pathway), and then a time was allowed during which the subject could train to feel these sensations;

2) Feel the KMI: the second step requests the participant to progressively reduce her/his muscular activity while focusing on the sensations perceived during the first step, in order to progressively obtain a KMI. This step allows the participant to concentrate on the feeling of activation of the peripheral pathway preceding each movement while having a visual feedback on his muscular activity thanks to an EMG electrode positioned on the forearm [19];

3) Implement the KMI: in the third step, the subject knows how to perform a KMI when she/he wants but needs to practice in order to be able to perform a KMI when the system instructs him to do it. The participant is placed in front of the Grasp-IT interface and is free to perform the KMI when the light turns green.

Members of the Trial-\&-Error group practiced the task by repeating KMIs. They were required to complete three sessions consisting of 20,30 and 20 KMIs respectively. A feedback was delivered as in a normal series based on the calibration of the first phase (Sec. II-D). Between the different runs, breaks similar to those performed by the Guided group were provided.

\section{Participants}

37 right-handed healthy subjects (15 females; aged 26.2 years old; STD $=10.42$ ) were recruited for this study. All the participants were novice in $\mathrm{BCI}$ and did not know what a KMI task was before starting the experiment. The education level of subjects is on average 3.29 years $(\mathrm{STD}=3.1)$ post high-school diploma and ensures that all subjects are able to understand the instruction during the experiment. The subjects had no medical history that could have influenced the task (i.e., diabetes, antidepressant treatment, or neurological disorders). The experiment follows the statements of the WMA declaration of Helsinki on ethical principles for medical research involving human subjects [20] and all participants signed an informed consent approved by the local ethical committee of Inria (COERLE, approval number: 2016-011/01).

\section{Experimental procedure}

Each participant took part in one session of 105 min divided in six parts: (1) Fill in the first questionnaires (15 min); (2) EEG cap installation (20 min); (3) First phase of the experiment (included a calibration and a testing steps) during which participants from both groups could interact with the BCI (Called Phase 1 = Calibration 1 and Test 1) (15 min); (4) Learning phase (the Guided group and the Trial-\&-Error group) (25 min); (5) Second phase of BCI use (Called Phase $2=$ Calibration 2 and Test 2) to assess the impact of the two different learning approaches on BCI performance (15 min); (6) Fill the postexperiment questionnaires $(15 \mathrm{~min})$ and uninstallation.

During the BCI use, the calibration step, similar for the two conditions, enabled the collection of data for both classes (right hand KMI and resting state) and the test step provided opportunity for the participant to interact with the Grasp-IT interface and become familiar with the cues and feedback provided by the visual environment (step called Test 1). Due to a breakdown, several subjects (S1, S2 and S14 for the Guided group and S7 for the Trial-\&-Error group) performed 25 tests during the calibration step of phase 1 .

During Phases 1 and 2 (Fig. 1A), all subjects were seated in front of a screen showing the non-immersive virtual environment of Grasp-IT composed of the traffic light and a virtual right hand (Fig. 1B). The whole step consisted of one run with 40 trials. During each trial, users were invited to perform the KMI of grasping continuously during 4 seconds, as soon as the light turned green and while it remained so. The rest condition was similarly indicated by the red light (Fig. 1C), lasting 6 seconds. Then, the orange light along with the red one, lasting 2 seconds, warned the subject that the KMI would start soon to prevent motor preparation by anticipation by the end of the red light period.

\section{E. Questionnaires}

Before Phase 1, the first round of non-standardized questionnaires (Mean time to fill them $=15 \mathrm{~min}$ ) aimed at finding out:

- pre-test perception of experience of using a BCI, in the form of 6 pairs of contrasting words (e.g., Painful/Pleasant, Anguishing/Reassuring, Discouraging/Motivating) to be assessed using 7-point Likert's scales;

- habits in terms of use of digital tools, video games but also in terms of sports, music or manual activities via 4- and 6-point Likert's scales and frequency scales;

- basic demographic data: age, gender, last degree obtained and current socio-professional category.

These questionnaires allowed us to verify the absence of significant differences between our two groups.

After Phase 2, a second round of standardized and nonstandardized questionnaires were completed (Mean time to fill them $=15 \mathrm{~min}$ ) to assess: 
- post-test perception of experience of BCI use, through the same 6 pairs of contracting words than for the pre-test;

- user feedbacks on relevance in the interface functioning and design;

- user experience through two questionnaires: the SUS [21] and the Attrakdiff (simplified french version) [22], which are two standardized questionnaires frequently used in HumanComputer Interaction to assess the perceived usability and pragmatic and hedonistic aspects of an interface just after its use.

These questionnaires allowed us (i) to check that Grasp-IT has worked well regardless of who is using it, (ii) to assess the user experience from different angles and also (iii) to study possible correlations between this UX and the BCI performance.

\section{F. Electrophysiological recordings}

EEG signals were recorded through the OpenViBE software platform with a Biosemi Active Two 32-channel EEG system. In accordance with the international 10-20 system, the EEG was recorded from 32 sites localized around the sensorimotor area. The selected electrodes are $F C_{5}, F C_{3}, F C_{1}, F C_{z}, F C_{2}$, $F C_{4}, F C_{6}, C_{5}, C_{3}, C_{1}, C_{z}, C_{2}, C_{4}, C_{6}, C P_{5}, C P_{3}, C P_{1}, C P_{z}$, $C P_{2}, C P_{4}, C P_{6}, P_{3}, P_{1}, P_{z}, P_{2}, P_{4}, \mathrm{PO}_{3}, \mathrm{PO}_{z}, \mathrm{PO}_{4}, O_{1}, O_{z}$, $\mathrm{O}_{2}$. An external electromyogram (EMG) electrode was used during the Feel the KMI and in order to verify that there was no movement during the KMI task.

\section{G. Online classification}

During this experiment, we compute online classification accuracy with the OpenViBE software to provide a neurophysiological feedback during the test step for both phases 1 and 2. After the calibration step, the recorded EEG signals were band-passed using a 5th-order Butterworth filter between 8 and $30 \mathrm{~Hz}$. Each trial was segmented into 3.5s-windows during the KMI task time and a rest time (Fig. 1C) starting respectively 0.5 seconds after the green light signal and 2.5 seconds after the red light signal. For each session, we collected 40 trials for both mental states (KMI and resting state). The training was performed using a CSP filter and a linear discriminant analysis (LDA). The classifier was trained to discriminate the two classes from 1s-windows with a sliding window of 0.0625 $\mathrm{s}$ during the $3.5 \mathrm{~s}$ of the EEG segments. Thus, for each trial and each class we have 40 patterns leading to 40 predictions. Performance Scores are the recall of each class (Fig. 2 and 3).

For the test step, an online classification was performed on 40 trials using the CSP filters and the LDA previously calculated from the calibration step $(1 \mathrm{~s}$ for each epoch according to a sliding window of $0.0625 \mathrm{~s}$ ). The feedback is only based on the recall of the MI class i.e., the number of correct classification (Fig. 2). The water jet as feedback is only given when the recall is over 20/40 and is then proportional to the recall. In order to verify the BCI consistency, we computed the recall for each class (KMI and resting state) (Fig. 3).

\section{H. Time-Frequency Analysis}

All offline analyses were performed using the EEGLAB toolbox 14.1 [24] and MATLAB 2016a. Raw EEG data were resampled at $256 \mathrm{~Hz}$, high-pass filtered at $0.5 \mathrm{~Hz}$ using a FIR filter and divided in 8 seconds epochs corresponding to 1 seconds before and 7 seconds after the motor task for each run. Then, a baseline was defined 2 seconds before each trial, hence a specific baseline was chosen for the two sessions. To analyze the differences between each group of participants (Guided vs Trial-\&-Error) and different phases of the experiment (prelearning and post-learning), we performed an event-related spectral perturbation (ERSP) between 8-35 Hz. We compute the ERSPs using the gain model approach which is equivalent to the band power method [4]. In this model, the ERSP at each time-frequency point is divided by the average spectral power in a 2 s pre-stimulus baseline period for each frequency bands. Then, a log-transformed ERSP measure was used to highlight our results (Eq. 1). The mean ERSP for frequency $f$ and time point $t$ is defined as:

$$
E R S P_{l o g}(f, t)=10 \log _{10}\left(E R S P_{\%}(f, t)\right)
$$

\section{RESUlts}

\section{A. Balance between the two groups of subjects}

Age, level of education, gender, sports and manual activities, were controlled to ensure a sufficient diversity of participants in both groups and a comparison of the effect of the two learning strategies. No significant differences (Fisher t-test, all p-values $>0.1$ ) were observed between the two panels. Before starting, participants in both groups reported that they were neither stressed nor excited. They had a neutral emotional perception of the experience (BCI-UX pre-test). In addition, their average BCI performance during the Phase 1 in terms of total number of water jets or classification score (Fig. 2) did not show statically significant differences between the two groups (t-student; pvalue $>0.1)$. In addition, there is also no significant difference in terms of ERD or ERS during the KMI (permutation test; $p$ $>0.05 ; 2,000$ permutations). It is important to note that both groups perform well from the outset. More than $50 \%$ of the subjects succeed in powerful jets until the 20th trial, then their performance progressively decreases, probably due to fatigue (Fig. 4).

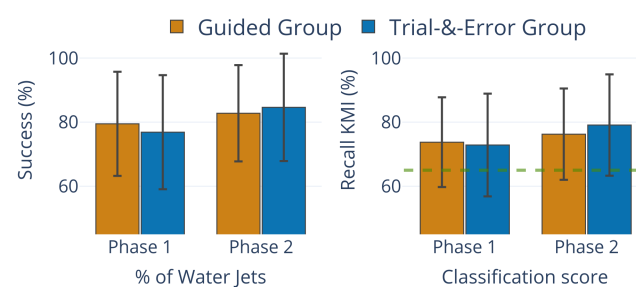

Fig. 2. Diagrams representing the percentage of all the water jets (left side) and the recall for KMI class (right side) related to the classifier during phase 1 and phase 2 for the Guided group and the Trial-\&-Error group. The dashed line (in green) indicates the chance level (65\%) according to the number of trials and the class [23]. 

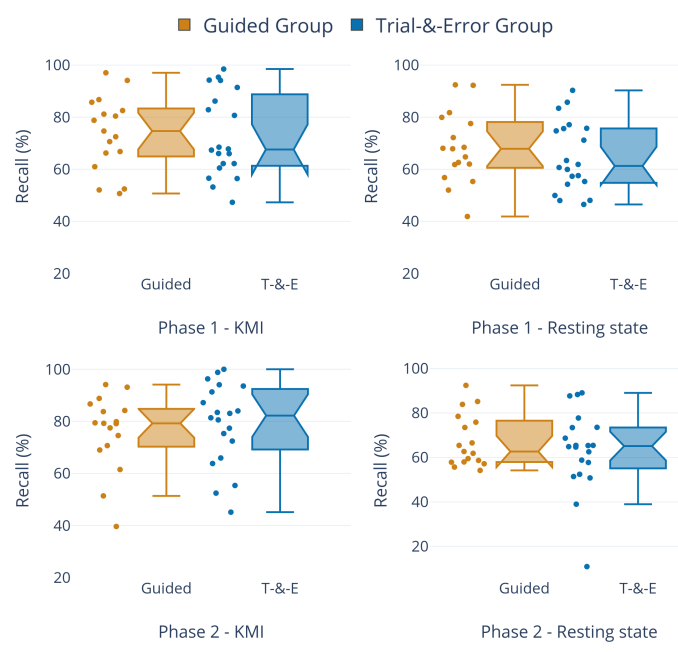

Fig. 3. Boxplots showing the coherence of the classifier accuracy for each group of participants (Guided in blue and Trial-\&-Error in orange). Accuracy was calculated for the KMI (left side) and resting state (right side) during both phases 1 and 2 .

\section{B. BCI performance}

After their learning phase (i.e., during Phase 2), there is no statistically significant difference (t-student; $p$-value $>$ 0.1 ) in terms of mean number of water jets obtained and mean score of BCI performance between the two groups of participants (Guided vs Trial-\&-Error) (Fig. 2 and 3). The subjects' mean performances between phase 1 and phase 2 are strongly correlated, whatever the condition tested (Trial$\&$-Error group pearson $=0.576, \mathrm{p}$-value $<0.01$; Guided group pearson $=0.638$, p-value $<0.01)$. This indicates that the tested learning procedures did not degrade the subjects' abilities who performed well on Test 1 . On the other hand, the absence of statistical significance does not mean that there are no differences. Indeed, the groups of participants both slightly

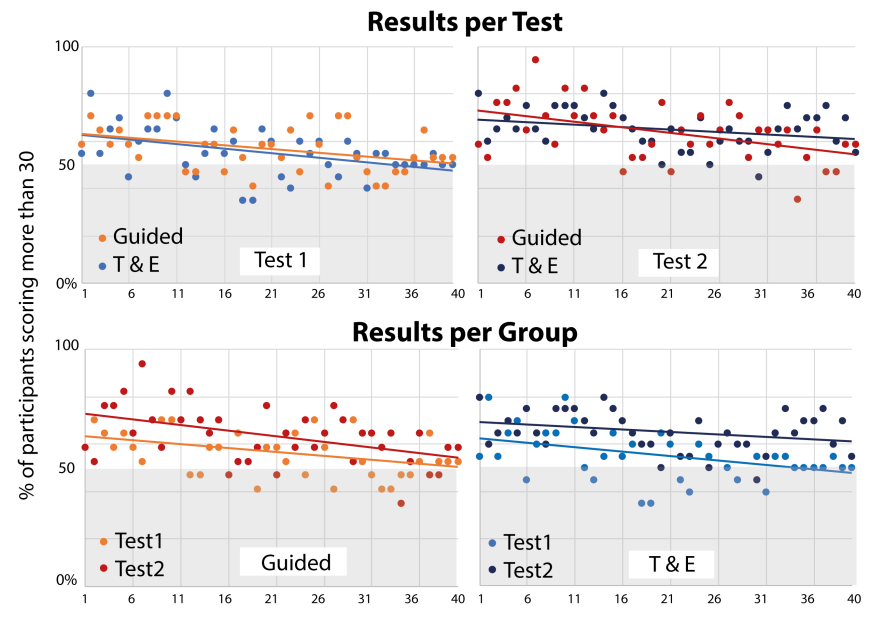

Fig. 4. Percentage of participants achieving high performance over the 40 tests per session. A point in the shaded area indicates that less than $50 \%$ of the subjects perform highly. progress from phase 1 to phase 2 (Fig. 2) (t-student; p-value $>$ 0.05). The number of participants who scored more than $30 / 40$ in each trial increased by $5 \%$ for the Trial-\&-Error group and $7 \%$ for the Guided group on average (Fig. 4; t-student; p-value $<0.05$ ). However, this slight progress does not seem to be explained in the same way. Participants in the Trial-\&-Error group manage to maintain KMI throughout the Test 2, thus producing a little more powerful jets at the end of the session compared to their Test 1 (Fig. 4); while guided subjects succeed more often than the previous group of high-powered jets during test 2, but their results remained uncertain and declined over the trials (Fig. 4).

\section{ERD and ERS modulations during the KMI}

The time-frequency analysis (Fig. 5 and 6) shows the ERD and ERS modulations for the KMI task before learning (Phase 1) and after learning (Phase 2) for both groups (Trial-\&-Error and Guided). There is a difference between the calibration and testing steps for Phases 1 and 2 in all participants (Trial-\&-Error and Guided groups). Indeed, during the calibration, an ERD is observed all along the KMI, mostly in the mu frequency band $(7-13 \mathrm{~Hz})$ whereas the ERD during the test step is extended after the end of the KMI. It is not surprising since at this time a feedback was given to the participant. Visual feedback visualization indeed supports ERD generation in the mu and beta bands over the motor cortex [25].

The evolution of the ERD modulations obtained after learning for each group of participants progress more in the guided group. This is particularly relevant for the test step where no change in the Trial-\&-Error group was observed (Fig. 5), whereas the guided group presents a stronger activity in the mu band after learning (Fig. 6). As the ERD phase reflects an activation of the motor cortex [26], this stronger ERD would suggest a better control of the KMI task after a guided learning. However, we cannot exclude that others as yet unknown factors explain this difference between the two groups.

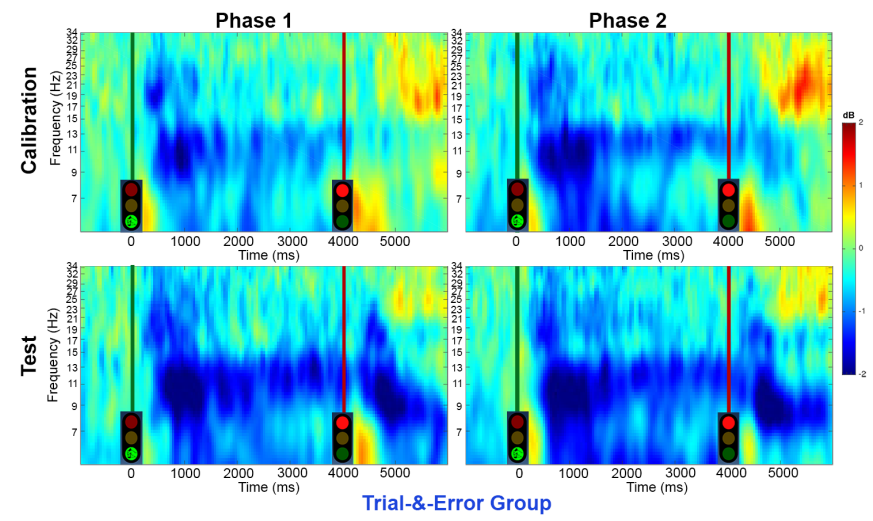

Fig. 5. Time-frequency grand average analysis $(n=20)$ corresponding to an event-related spectral perturbation (ERSP) for the Phase 1 (Pre-Learning) and Phase 2 (Post-Learning) for the Trial-\&-Error group for electrode $C_{3}$. 


\section{BCI usability}

From a functional point of view, BCI performances among all subjects (Fig. 2 and 3) indicate that Grasp-IT detects on average $75 \%$ of the KMIs, which is significant $(\mathrm{p}>0.01)$ considering the number of trials for the test step (40 trials for KMI class and 40 trials for resting state class) [23]. Moreover, both the KMI class and the rest class are detected by Grasp-IT (Fig. 3).

From the user's point of view, although considered a bit tiring, all participants reported a positive learning experience with Grasp-IT whatever the learning strategy provided (Trial\&-Error or Guided). All considered the Grasp-IT BCI as usable and attractive due to its pragmatic and hedonistic qualities, which are reflected in the results of the SUS and Attrakdiff surveys (Fig. 7).

\section{E. Learning Experience}

We investigated whether there was a difference in perception among participants based on the learning method provided. $75 \%$ of the participants in the Trial-\&-Error group found the KMI task exhausting, compared to $53 \%$ in the Guided group. In addition, the Trial-\&-Error group participants also found the system significantly more inconsistent than those in the guided group (1.63 vs. 1.23, p-value < 0.05). Moreover, there are correlations between the mean performance of the subjects in the Trial-\&-Error group with the fact that they found (i) the system unpredictable (rho Spearman $=0.512$, p-value $<0.05$ ), (ii) the experience motivating (rho Spearman $=470 \mathrm{p}$-value $<0.05$ ) and (ii) the traffic light as helpful in knowing when to perform the task (rho Spearman $=0.466$, p-value $<0.05$ ). These correlations are absent for the guided group.

The guided group saw their learning experience in a much more positive way from the motivation point of view. The higher their performance, (i) the more the guided subjects declare to be able to use the system frequently in the future (rho Spearman $=0.648$, p-value $<0.005$ ), (ii) the more they find Grasp-IT easy and simple to use (rho Spearman Easy = 0.671 , p-value $<0.005$, rho Spearman Simple $=0.483$, p-value $=0.05$ ) and (iii) the less they find the system inconsistent (rho

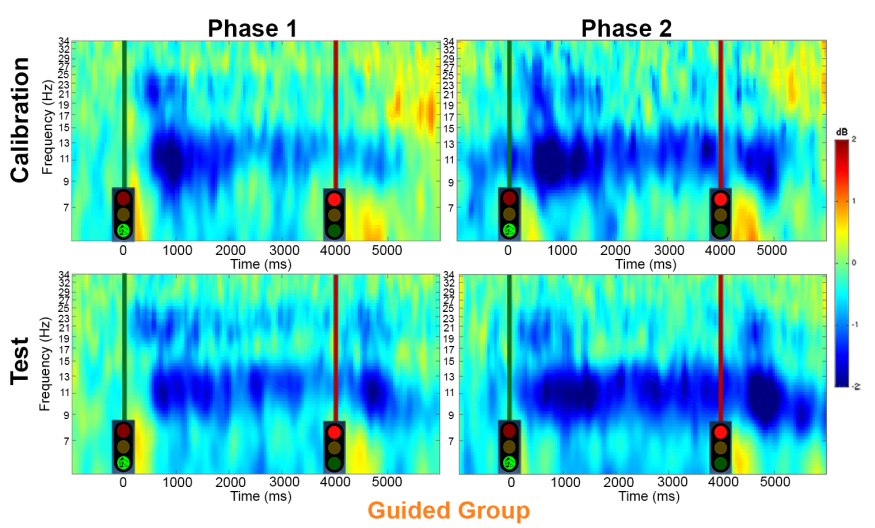

Fig. 6. Time-frequency grand average analysis $(n=17)$ corresponding to an event-related spectral perturbation (ERSP) for the Phase 1 (Pre-Learning) and Phase 2 (Post-Learning) for the Guided group for electrode $C_{3}$.

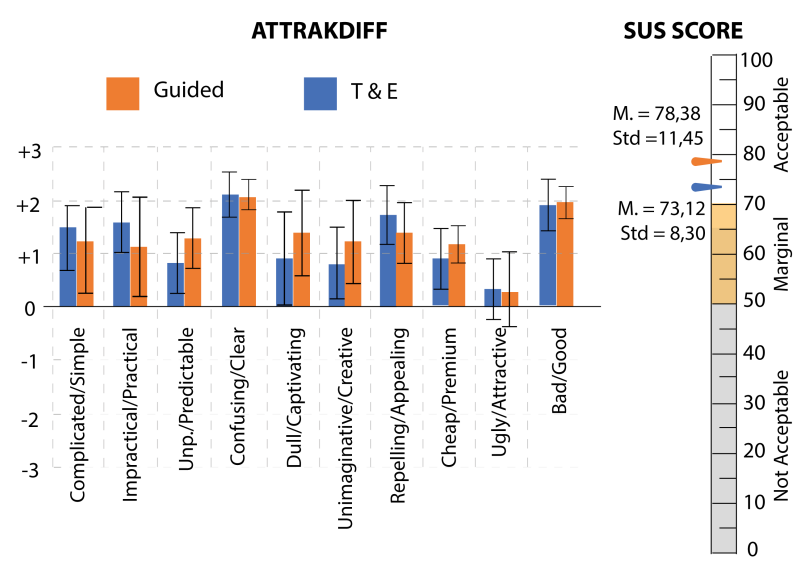

Fig. 7. Mean values obtained with the simplify Attrakdiff survey (French version [22]) and with the SUS for the two groups of Grasp-IT users.

Spearman $=0.585, \mathrm{p}$-value $<0.01)$. All these correlations are absent for the Trial-\&-Error group.

\section{DISCUSSION}

This study highlighted both usability and the relevance of design choices of the Grasp-IT BCI as an appropriate learning environment to support KMI's task. Nevertheless, whether in terms of BCI performance or ERD/ERS modulations, few differences were observed according to both learning approaches. The following subsections detail and discuss these observations.

\section{A. Strengths and weaknesses of the learning approaches}

Combined to the Grasp-IT BCI, the two KMI learning approaches assessed in this study are quite different and each has both advantages and limitations. Learning by trial-anderror requires the participant to build her/his own strategy throughout a repetitive process. It is based on the premise that a learner will be able on her/his own to make inferences from perceived feedbacks. The plebiscite of the Trial-\&-Error group for the Grasp-IT elements designed to support the KMI implementation, combined with the fact that all subjects were able to perform well during the two phases despite fatigue, validates the design choices of the Grasp-IT environment. However, this group (Trial-\&-Error) often finds that the interface is inconsistent, which is not the case for the Guided group. This could indicates that learners of the Trial-\&-Error group misunderstood the cause and effect relationship between the jet power produced by the system and the KMI they believed successful (or not). This result indicates that using a trial-anderror approach can have an impact on how a learner could figure out her/his KMI, and consequently, influence her/his KMI-based BCI experience.

The guided KMI learning focuses on the notion of progressiveness from a real movement to the integration of the kinesthetic sensations related to that movement. Some clues indicate that the KMI task seems to be integrated by the participants of this group (ERD/ERS modulations, BCI-User experience, etc.). Grasp-IT combined with human support to 
learn KMI progressively seems to enable the subjects to perceive their successes well and to feel more confident to use the BCI again independently. This combined approach were therefore considered to be more "reliable" and acceptable by these subjects (Fig. 7) which is very important for a BCI [11].

\section{B. Limitation and Perspectives}

However, the performance results do not allow firm conclusions to be drawn about the efficacy of the compared learning approaches. Three different explanations, opening new perspective, could be proposed. First, the learning processes were not sufficiently long-term, or deep enough, to ensure that the KMI competencies were integrated into a mental scheme. A recent study [27] showed that significant effects of learning on ERD phase or BCI performance were not observed before three weeks, which could explain the results obtained (Fig. 2, 5 and 6). Second, the overall duration of the experiment was in total quite long ( $>2$ hours). Some participants, exhausted or unable to maintain their attention, might under-performed during the test or the calibrations steps in the post-learning phase, not because they did not learn, but rather because the length of the experience would have influenced the success of one of the two steps (calibration or test).

\section{CONClusion}

Although further work on the effectiveness of the two learning methods remains to be done, the Grasp-It design choices thought to support the KMI implementation are validated. The approach that combines the Grasp-IT paradigm with progressive and guided KMI learning seems more reliable and more acceptable for the participant, particularly in terms of supporting their motivation and KMI task understanding, so many benefits that are particularly required in stroke recovery protocols for example. Based on this ongoing study, we will proceed the experiment with various scenarios of learning sessions over a longer period of time, and also with subjects who were initially poor performers or even brain damaged.

\section{REFERENCES}

[1] K. Ridderinkhof and M. Brass, "How kinesthetic motor imagery works: a predictive-processing theory of visualization in sports and motor expertise," J Physiol Paris, vol. 109, no. 1, pp. 53-63, 2015.

[2] A. Guillot, C. Collet, V. A. Nguyen, F. Malouin, C. Richards, and J. Doyon, "Brain activity during visual versus kinesthetic imagery: an fmri study.” Hum Brain Mapp, vol. 30, no. 7, pp. 2157-2172, Jul 2009.

[3] S. Hétu, M. Gregoire, A. Saimpont, M.-P. Coll, F. Eugène, P.-E. Michon, and P. Jackson, "The neural network of motor imagery: An ale metaanalysis," Neuroscience and biobehavioral reviews, vol. 37, 042013.

[4] G. Pfurtscheller and F. H. Lopes da Silva, "Event-related EEG/MEG synchronization and desynchronization: basic principles," Clin Neurophysiol, vol. 110, no. 11, pp. 1842-57, Nov 1999.

[5] G. Pfurtscheller, "Functional brain imaging based on ERD/ERS," Vision Research, vol. 41, no. 10-11, pp. 1257 - 1260, 2001.

[6] C. Lindig-Leon, L. Bougrain, and S. Rimbert, "Alpha rebound improves on-line detection of the end of motor imageries," in IEEE EMBS Neural engineering conference, Montpellier, France, Apr. 2015.

[7] M. A. Cervera, S. R. Soekadar, J. Ushiba, J. d. R. Millán, M. Liu, N. Birbaumer, and G. Garipelli, "Brain-computer interfaces for poststroke motor rehabilitation: a meta-analysis," Annals of Clinical and Translational Neurology, vol. 5, no. 5, pp. 651-663, 2018.
[8] L. Mcavinue and I. Robertson, "Motor imagery: a multidimensional ability," Journal of Mental Imagery, vol. 33, p. 99, 082010.

[9] M. Ahn, H. Cho, S. Ahn, and S. C.Jun, "User's self-prediction of performance in motor imagery brain-computer interface," Frontiers in Human Neuroscience, vol. 59, no. 12, 2018.

[10] G. Morone, I. Pisotta, F. Pichiorri, S. Kleih, S. Paolucci, M. Molinari, F. Cincotti, A. Kübler, and D. Mattia, "Proof of principle of a braincomputer interface approach to support poststroke arm rehabilitation in hospitalized patients: Design, acceptability, and usability," Archives of Physical Medicine and Rehabilitation, vol. 96, no. 3, Supplement, pp. S71 - S78, 2015, the 5th International BCI Meeting.

[11] C. Jeunet, E. Jahanpour, and F. Lotte, "Why standard brain-computer interface (BCI) training protocols should be changed: an experimental study," Journal of Neural Engineering, vol. 13, no. 3, 2016.

[12] F. Lotte, "On the need for alternative feedback training approaches for bci," Berlin Brain-Computer Interface Workshop, 092012.

[13] S. Rimbert, L. Bougrain, R. Orhand, J. Nex, S. Gaborit, and S. Fleck, "Grasp'it : une interface cerveau-ordinateur pour l'amélioration de l'apprentissage d'une tâche d'imagination motrice kinesthésique," in 29ème conférence francophone sur l'IHM, Poitiers, France, Aug. 2017, p. 2 p.

[14] S. Rimbert, N. Gayraud, L. Bougrain, M. Clerc, and S. Fleck, "Can a subjective questionnaire be used as brain-computer interface performance predictor?" Frontiers in Human Neuroscience, vol. 12, p. 529, 2019.

[15] S. M. Freeman, S. Itthipuripat, and A. R. Aron, "High working memory load increases intracortical inhibition in primary motor cortex and diminishes the motor affordance effect," Journal of Neuroscience, vol. 36, no. 20, pp. 5544-5555, 2016.

[16] A. Vuckovic and B. Osuagwu, "Using a motor imagery questionnaire to estimate the performance of a brain-computer interface based on object oriented motor imagery," Clin Neurophysiol, vol. 8, no. 124, pp. 1586-95, 2013.

[17] S. Kleih, A. Riccio, D. Mattia, V. Kaiser, E. Friedrich, R. Scherer, G. Müller-Putz, C. Neuper, and A. Kübler, "Motivation influences performance in smr-bci," Proceedings of the Fifth International Brain-Computer Interface Conference 2011, 012011.

[18] L.-F. Tan, Z. Dienes, A. Jansari, and S.-Y. Goh, "Effect of mindfulness meditation on brain-computer interface performance," Consciousness and Cognition, vol. 23, pp. $12-21,2014$.

[19] V. V. Nikulin, F. U. Hohlefeld, A. M. Jacobs, and G. Curio, "Quasimovements: A novel motor-cognitive phenomenon," Neuropsychologia, vol. 46, no. 2, pp. $727-742,2008$.

[20] A. World Medical, "World medical association declaration of helsinki: ethical principles for medical research involving human subjects." $J$ Postgrad Med, vol. 48, no. 3, pp. 206-208, Jul-Sep 2002, kIE: KIE Bib: human experimentation.

[21] J. Brooke, "Sus - a quick and dirty usability scale." CRC Press, 2006.

[22] C. Lallemand, V. Koenig, G. Gronier, and R. Martin, "Création et validation d'une version française du questionnaire attrakdiff pour l'évaluation de l'expérience utilisateur des systèmes interactifs," European Review of Applied Psychology, vol. 65, pp. 239-252, 092015.

[23] C. Jeunet, S. Debener, F. Lotte, J. Mattout, R. Scherer, and C. Zich, Mind the Traps! Design Guidelines for Rigorous BCI Experiments. Nam, S and Nijholt, A and Lotte, F, 012018.

[24] A. Delorme and S. Makeig, "EEGLAB: an open source toolbox for analysis of single-trial eeg dynamics including independent component analysis," Journal of Neuroscience Methods, vol. 134(1), pp. 9-21, 2004.

[25] C. Neuper, R. Scherer, S. Wriessnegger, and G. Pfurtscheller, "Motor imagery and action observation: Modulation of sensorimotor brain rhythms during mental control of a brain-computer interface," Clinical neurophysiology : official journal of the International Federation of Clinical Neurophysiology, vol. 120, pp. 239-47, 022009.

[26] C. Neuper, M. Wörtz, and G. Pfurtscheller, "Erd/ers patterns reflecting sensorimotor activation and deactivation," in Event-Related Dynamics of Brain Oscillations, ser. Progress in Brain Research, C. Neuper and W. Klimesch, Eds. Elsevier, 2006, vol. 159, pp. $211-222$.

[27] M. Jianjun and B. He, "Exploring training effect in 42 human subjects using a non-invasive sensorimotor rhythm based online bci," Frontiers in Human Neuroscience, vol. 13, 042019. 